九州大学学術情報リポジトリ

Kyushu University Institutional Repository

On the Local Asymptotic Behavior of the Likelihood Function for Meixner Lévy Processes under High-Frequency Sampling

Kawai, Reiichiro

Department of Mathematics, University of Leicester

Masuda, Hiroki

Graduate School of Mathematics, Kyushu University

http://hdl. hand le. net/2324/17916

出版情報: MI Preprint Series. 2010-26，2010-08-08. Faculty of Mathematics, Kyushu University バージョン：

権利関係 : 


\section{Preprint Series}

Kyushu University

The Global COE Program

Math-for-Industry Education \& Research Hub

On the local asymptotic behavior of the likelihood function for Meixner Lévy processes under high-frequency sampling

\section{Reiichiro Kawai \& Hiroki Masuda}

MI 2010-26

( Received August 8, 2010 )

Faculty of Mathematics

Kyushu University

Fukuoka, JAPAN 


\title{
On the Local Asymptotic Behavior of the Likelihood Function for Meixner Lévy Processes under High-Frequency Sampling
}

\author{
REIICHIRO KAWAI* AND HIROKI MASUDA $^{\dagger}$
}

\begin{abstract}
We discuss the local asymptotic behavior of the likelihood function associated with all the four characterizing parameters $(\alpha, \beta, \delta, \mu)$ of the Meixner Lévy process under high-frequency sampling scheme. We derive the optimal rate of convergence for each parameter and the Fisher information matrix in a closed form. The skewness parameter $\beta$ exhibits a slower rate alone, relative to the other three parameters free of sampling rate. An unusual aspect is that the Fisher information matrix is constantly singular for full joint estimation of the four parameters. This is a particular phenomenon in the regular high-frequency sampling setting and is of essentially different nature from low-frequency sampling. As soon as either $\alpha$ or $\delta$ is fixed, the Fisher information matrix becomes diagonal, implying that the corresponding maximum likelihood estimators are asymptotically orthogonal.
\end{abstract}

Keywords: High-frequency sampling, Lévy process, local asymptotic normality, Meixner process, Fisher information matrix.

2010 Mathematics Subject Classification: 60G51, 62E20.

\section{Introduction and Preliminaries}

The local asymptotic normality (LAN, for short) property is a vital concept in asymptotically optimal statistical analyses. In short, the LAN property is defined through the following locally asymptotically quadratic structure of a likelihood ratio

$$
L_{n}\left(\theta+R_{n} u\right)-L_{n}(\theta)=\left\langle u, \mathscr{H}_{n}(\theta)\right\rangle-\frac{1}{2}\langle u, \mathscr{I}(\theta) u\rangle+o_{\mathbb{P}_{\theta}}(1)
$$

for each $u$, where $\mathbb{P}_{\theta}$ is a probability measure associated with the parameter $\theta$, where $\left\{R_{n}\right\}_{n \in \mathbb{N}}$ is a sequence of nonrandom positive definite matrices tending to 0 in norm, where $\left\{\mathscr{H}_{n}(\theta)\right\}_{n \in \mathbb{N}}$ is a sequence of random vectors converging in law to $\mathscr{N}(0, \mathscr{I}(\theta))$ under $\mathbb{P}_{\theta}$, and where $\mathscr{I}(\theta)$ is a nonnegative definite deterministic matrix, called the Fisher information matrix. Once the identity $(1.1)$ is confirmed with nonsingular $\mathscr{I}(\theta)$, one can formulate asymptotic optimality of estimation and testing hypothesis in terms of $\mathscr{H}_{n}(\theta)$. (See Le Cam [7], Le Cam and Yang [8], and van der Vaart [14] for a systematic account of the LAN theory.)

In this article, we discuss the local asymptotic behavior of the likelihood function associated with the fourparameter Meixner Lévy process observed under high-frequency sampling scheme. The Meixner process has been recognized as a successful class of Lévy processes for the purpose of practical modeling, such as mathematical finance and possibly turbulence, as well as of sufficient theoretical interest. We begin with some fundamental facts of the Meixner process with the most popular parametrization. (We refer the reader to [2, 13] for general details, and also [3, 6] for numerical aspects of the Meixner process.) The Meixner distribution, denoted by Meixner $(\alpha, \beta, \delta, \mu)$, is infinitely divisible and selfdecomposable, and admits a probability density

$$
x \mapsto \frac{(2 \cos (\beta / 2))^{2 \delta}}{2 \pi \alpha \Gamma(2 \delta)} \exp \left[\frac{\beta}{\alpha}(x-\mu)\right]\left|\Gamma\left(\delta+i \frac{x-\mu}{\alpha}\right)\right|^{2}, \quad x \in \mathbb{R},
$$

where $\alpha>0,|\beta|<\pi, \delta>0, \mu \in \mathbb{R}$. When $\beta=0$, the distribution is symmetric around $\mu$. We write

$$
\theta:=(\alpha, \beta, \delta, \mu) \in \Theta,
$$

\footnotetext{
This version: August 8, 2010.

*Email Address: reiichiro.kawai@gmail.com. Postal Address: Department of Mathematics, University of Leicester, Leicester LE1 7RH, UK.

$\dagger$ Email Address: hiroki@math.kyushu-u.ac.jp. Postal Address: Graduate School of Mathematics, Kyushu University, Motooka 744, Nishi-ku, Fukuoka 819-0395, Japan.
} 
the parameter space $\Theta$ being a bounded convex domain satisfying

$$
\Theta^{-} \subset\left\{(\alpha, \beta, \delta, \mu) \in \mathbb{R}^{4}|\alpha>0,| \beta \mid<\pi, \delta>0, \mu \in \mathbb{R}\right\} .
$$

The Lévy measure of $\operatorname{Meixner}(\alpha, \beta, \delta, \mu)$ admits the Lebesgue density

$$
g(z ; \theta):=\delta \frac{\exp (\beta z / \alpha)}{z \sinh (\pi z / \alpha)}, \quad z \in \mathbb{R}_{0}:=\mathbb{R} \backslash\{0\} .
$$

Let $\left\{X_{t}: t \geq 0\right\}$ be a Lévy process satisfying

$$
\mathscr{L}\left(X_{1}\right)=\operatorname{Meixner}(\alpha, \beta, \delta, \mu),
$$

which we call a Meixner (Lévy) process, which is of infinite variation. We denote by $\mathbb{P}_{\theta}$ the distribution of $X$ associated with the parameter $\theta \in \Theta$ and by $\mathbb{E}_{\theta}$ the expectation taken under the probability measure $\mathbb{P}_{\theta}$. In what follows, every stochastic asymptotics is taken under $\mathbb{P}_{\theta}$. The characteristic function of $\mathscr{L}\left(X_{1}\right)$ is given in closed form by

$$
\mathbb{E}_{\theta}\left[e^{i y X_{1}}\right]=e^{i y \mu}\left(\frac{\cos (\beta / 2)}{\cosh ((\alpha y-i \beta) / 2)}\right)^{2 \delta}, \quad y \in \mathbb{R},
$$

which implies that the Meixner distribution possesses the reproducing property, and that for each $c>0$ and $t>0$,

$$
\mathscr{L}\left(c\left(X_{t}-t \mu\right)\right)=\operatorname{Meixner}(c \alpha, \beta, t \delta, 0) .
$$

One of the remarkable properties of the Meixner process is its asymptotic behavior with respect to observation time, just like normal inverse Gaussian processes, tempered stable processes of Rosiński [11] and layered stable processes of Houdré and Kawai [4]. On the one hand, over short time intervals, it approximates a stable process; as $h \downarrow 0$, a scaled Meixner process

$$
\left\{\frac{1}{h \alpha \delta}\left(X_{h t}-h t \mu\right): t \geq 0\right\}
$$

tends to a standard Cauchy (Lévy) process, where the convergence is in the weak sense of random processes in the space of càdlàg functions from $[0,+\infty)$ into $\mathbb{R}$ equipped with the Skorohod topology. (See also Lemma 3.1 below.) In a long time frame, on the other hand, it is close to a Brownian motion; as $h \uparrow+\infty$, another scaled Meixner process

$$
\left\{\frac{\cos (\beta / 2)}{\alpha} \sqrt{\frac{2}{h \delta}}\left(X_{h t}-h t\left(\mu+\alpha \delta \tan \frac{\beta}{2}\right)\right): t \geq 0\right\}
$$

approaches to the standard Brownian motion. (These can be proved in a similar manner to [4, 11].) The stable-type and Gaussian-type behaviors above have long been considered to be very appealing in various applications.

\section{Local Asymptotic Behavior of Likelihood Function}

Consider the sample $\left(X_{t_{n, 1}}, X_{t_{n, 2}}, \ldots, X_{t_{n, n}}\right)$ observed at equidistant observation points in time,

$$
t_{n, k}:=k \Delta_{n}, \quad k=1, \ldots, n,
$$

with a sequence $\left\{\Delta_{n}\right\}_{n \in \mathbb{N}}$ of positive stepsizes satisfying

$$
\Delta_{n} \downarrow 0 \quad \text { and } \quad n \Delta_{n} \uparrow \infty, \quad \text { as } n \uparrow+\infty .
$$

Let us state the main claim of this article. To maintain the flow, we defer the proof to Section 3.1

Theorem 2.1. The LAN property (1.1) holds for each $\theta \in \Theta$, with

$$
\begin{gathered}
R_{n}=\operatorname{diag}\left(\frac{1}{\sqrt{n}}, \frac{1}{\sqrt{n \Delta_{n}}}, \frac{1}{\sqrt{n}}, \frac{1}{\sqrt{n}}\right), \\
\mathscr{I}(\theta)=\left[\begin{array}{cccc}
\frac{1}{2 \alpha^{2}} & 0 & \frac{1}{2 \alpha \delta} & 0 \\
0 & \frac{\delta}{2 \cos ^{2}(\beta / 2)} & 0 & 0 \\
\frac{1}{2 \alpha \delta} & 0 & \frac{1}{2 \delta^{2}} & 0 \\
0 & 0 & 0 & \frac{1}{2 \alpha^{2} \delta^{2}}
\end{array}\right] .
\end{gathered}
$$


Observe that the Fisher information matrix (2.2) is singular, that is, $|\mathscr{I}(\theta)| \equiv 0$ for each $\theta \in \Theta$. Due to this fact, on the one hand, the conventional asymptotic optimality theory is not applicable to the full joint estimation of the four parameters. On the other hand, it is clear that the singularity is caused solely by the off-diagonal elements between $\alpha$ and $\delta$. Nevertheless, as soon as $\alpha$ or $\delta$ is fixed, the Fisher information matrix then reduces to $\mathbb{R}^{3 \times 3}$ and purely diagonal. This ensures that the maximum likelihood estimators are asymptotically independent.

Let us discuss the singularity issue for Meixner Lévy processes in terms of sampling scheme. First, it will shortly turn out (based on Lemma 3.2) that under low-frequency sampling with $\Delta_{n} \equiv \Delta>0$, the Fisher information matrix remains involved with infinite sums. It seems difficult to judge in an analytical manner whether the matrix is singular. (See also [3].) Next, it is worthwhile to compare with the continuous sampling setting, based upon the following result.

Proposition 2.2. Let $T>0$ and let $\theta_{k}:=\left(\alpha_{k}, \beta_{k}, \delta_{k}, \mu_{k}\right) \in \Theta, k=1,2$. The probability measures $\mathbb{P}_{\theta_{1}} \mid \mathscr{F}_{T}$ and $\mathbb{P}_{\theta_{2}} \mid \mathscr{F}_{T}$ are equivalent iff $\alpha_{1} \delta_{1}=\alpha_{2} \delta_{2}$ and $\mu_{1}=\mu_{2}$.

This proposition implies that singularity in studying likelihood becomes more noteworthy in the continuously observed case than in the high-frequency sampling case: in the latter case, the likelihood itself does exist for every admissible parameter values, while the Fisher information may be singular; in the former case, the likelihood itself may not exist. Especially what is interesting is that the location parameter $\mu$ is required to be fixed.

Let us next discuss the singularity issue for different classes of Lévy processes under high-frequency sampling. It is well known that a similar phenomenon is observed in the case of non-Gaussian stable Lévy process. Precisely, the joint maximum-likelihood estimation of the stability index and the scale parameter leads to a constantly singular Fisher information matrix. (See [1, 10], for example.) Inferring from this, we suspect that the singularity arises from every Lévy process whose short-range behavior can be approximated in law by a stable process with unknown stability index and scale parameter. Typical examples are tempered stable processes [11] and layered stable processes [4]. In this direction, the present setting of Meixner processes is not directly relevant since its short-time stability index is necessarily 1 (see Lemma 3.1 later). As observed in the Fisher information matrix (2.2), the singularity issue in our framework comes instead from $(\alpha, \delta)$.

In principle, unlike the low-frequency sampling case, the high-frequency sampling scheme yields different optimal rates of convergence for different characterizing parameters. There exist several case studies in the literature that address the joint LAN property for univariate Lévy processes. The most well known case is the scaled Wiener process with drift, $X_{t}=t \mu+\sigma W_{t}$, where the LAN property holds true for $(\mu, \sigma)$ at rate $\left(\sqrt{n \Delta_{n}}, \sqrt{n}\right)$ with a diagonal Fisher information matrix. In the case of inverse Gaussian subordinators or gamma processes, both of which are characterized by the two parameters $(\delta, \gamma)$, the LAN property holds true at rate $\left(\sqrt{n}, \sqrt{n \Delta_{n}}\right)$ with a diagonal Fisher information matrix. (See Masuda [9] for details.) More recently, the authors derive in [5] the LAN property for the normal inverse Gaussian (NIG) process, which is characterized by the four parameters $(\alpha, \beta, \delta, \mu)$. Again, the LAN property holds true at rate $\left(\sqrt{n \Delta_{n}}, \sqrt{n \Delta_{n}}, \sqrt{n}, \sqrt{n}\right)$ with a block-diagonal Fisher information matrix. Interestingly, the NIG process suffers no singularity issue, while, when suitably normalized, sharing the same short-range behavior of Cauchy type with the Meixner process.

Finally, let us note that the LAN property may be investigated, either singular or non-singular, only when the likelihood function are available in a sufficiently tractable form, such as (3.1) and (3.2). But, this is very rare. For example, the likelihood function for tempered stable processes is unknown in a closed form. Note also that the availability of an explicit likelihood function may not be enough. For example, without the reproducing property such as (1.3), even the explicit likelihood function is intractable in the high-frequency sampling framework. (A typical example is the generalized hyperbolic Lévy process.) This also ensures the importance of case studies.

\section{Proofs}

Throughout the proofs, we denote by $\Gamma(z)$ the Gamma function $\Gamma(z):=\int_{0}^{+\infty} x^{z-1} e^{-x} d x$, where $z$ is a complex number with a positive real part, denote by $\zeta$ the Riemann zeta function, that is, $\zeta(s):=\sum_{k=1}^{+\infty} k^{-s}, s>1$, and write $\gamma:=$ $\lim _{n \uparrow+\infty}\left(\sum_{k=1}^{n} k^{-1}-\ln n\right) \approx 0.5772$ for the Euler-Mascheroni constant.

\subsection{Proof of Theorem 2.1}

It holds by (1.3) that for each $n \in \mathbb{N}$,

$$
x_{n, k}:=X_{t_{n, k}}-X_{t_{n, k-1}} \sim \operatorname{Meixner}\left(\alpha, \beta, \Delta_{n} \delta, \Delta_{n} \mu\right), \quad k=1, \ldots, n .
$$


In view of this fact and the probability density function (1.2), define for each $n \in \mathbb{N}$ and $k=1, \ldots, n$,

$$
l_{n, k}(\theta):=2 \Delta_{n} \delta \ln \left(2 \cos \frac{\beta}{2}\right)-\ln (2 \pi \alpha)-\ln \Gamma\left(2 \Delta_{n} \delta\right)+\frac{\beta}{\alpha}\left(x_{n, k}-\Delta_{n} \mu\right)+\ln \left|\Gamma\left(\Delta_{n} \delta+i \frac{x_{n, k}-\Delta_{n} \mu}{\alpha}\right)\right|^{2} .
$$

Thanks to the stationarity and independence of increments of Lévy processes, the log-likelihood function to be maximized with discrete observations $\left\{X_{t_{n, k}}\right\}_{k=1, \ldots, n}$ is as simple as

$$
L_{n}(\theta):=\sum_{k=1}^{n} l_{n, k}(\theta)
$$

1) We begin with the local Cauchy approximation. We define a sequence $\left\{\varepsilon_{n, k}\right\}_{k=1, \ldots, n}$ of iid random variables by

$$
\varepsilon_{n, k}:=\varepsilon_{n, k}\left(\alpha, \delta, \mu, \Delta_{n}\right):=\frac{x_{n, k}-\Delta_{n} \mu}{\Delta_{n} \alpha \delta} \sim \operatorname{Meixner}\left(\frac{1}{\Delta_{n} \delta}, \beta, \Delta_{n} \delta, 0\right) .
$$

In the pathwise sense, the random variables $\left\{\varepsilon_{n, k}\right\}_{k=1, \ldots, n}$ depend on $\left(\alpha, \delta, \mu, \Delta_{n}\right)$ and is independent of $\beta$. In contrast, the law $\mathscr{L}\left(\varepsilon_{n, 1}\right)$ depends on $\left(\beta, \delta, \Delta_{n}\right)$ and is independent of $(\alpha, \mu)$. We can show that the law $\mathscr{L}\left(\varepsilon_{n, 1}\right)$ has the mean, the variance, the skewness and the kurtosis, respectively,

$$
\tan \frac{\beta}{2}, \quad \frac{1}{2 \Delta_{n} \delta \cos ^{2}(\beta / 2)}, \quad \sin \frac{\beta}{2} \sqrt{\frac{2}{\Delta_{n} \delta}}, \quad 3+\frac{2-\cos (\beta)}{\Delta_{n} \delta} .
$$

(See, for example, Grigelionis [2].) It then follows that as $n \uparrow+\infty$, the first four moments are of order $O(1), O\left(\Delta_{n}^{-1}\right)$, $O\left(\Delta_{n}^{-3 / 2}\right)$ and $O\left(\Delta_{n}^{-2}\right)$ if $\beta \neq 0$, while $0, O\left(\Delta_{n}^{-1}\right), 0$ and $O\left(\Delta_{n}^{-2}\right)$ if $\beta=0$.

We denote by the standard Cauchy distribution the infinitely divisible distribution with characteristic function $y \mapsto e^{-|y|}$ and the probability density function

$$
\phi(x):=\frac{1}{\pi\left(1+x^{2}\right)}, \quad x \in \mathbb{R} .
$$

The following lemma indicates that the random variables $\varepsilon_{n, k}$ act as suitably normalized increments.

Lemma 3.1. The law $\mathscr{L}\left(\varepsilon_{n, 1}\right)$ converges to the standard Cauchy distribution, as $n \uparrow+\infty$.

Proof. The claim can be deduced readily by observing that, for each $y \in \mathbb{R}$,

$$
\begin{aligned}
\mathbb{E}_{\theta}\left[e^{i y \varepsilon_{n, 1}}\right] & =\left(\frac{\cos (\beta / 2)}{\cosh \left(\left(y /\left(\Delta_{n} \delta\right)-i \beta\right) / 2\right)}\right)^{2 \Delta_{n} \delta} \\
& =\left(\frac{2}{e^{y /\left(2 \Delta_{n} \delta\right)}(1-i \tan (\beta / 2))+e^{-y /\left(2 \Delta_{n} \delta\right)}(1+i \tan (\beta / 2))}\right)^{2 \Delta_{n} \delta} \\
& \sim \begin{cases}\left(\frac{2}{1-i \tan (\beta / 2)}\right)^{2 \Delta_{n} \delta} e^{-y}, & \text { if } y>0, \\
\left(\frac{2}{1+i \tan (\beta / 2)}\right)^{2 \Delta_{n} \delta} e^{y}, & \text { if } y<0, \\
& \rightarrow e^{-|y|},\end{cases}
\end{aligned}
$$

as $n \uparrow+\infty$, with the help of the Lévy continuity theorem.

2) The likelihood function (3.2) in question is smooth in $\theta$. We can rewrite (3.1) as

$$
l_{n, k}(\theta)=2 \Delta_{n} \delta \ln [2 \cos (\beta / 2)]-\ln (2 \pi \alpha)-\ln \Gamma\left(2 \Delta_{n} \delta\right)+\Delta_{n} \beta \delta \varepsilon_{n, k}+\ln \left|\Gamma\left(\Delta_{n} \delta\left(1+i \varepsilon_{n, k}\right)\right)\right|^{2} .
$$

In our proof of Theorem 2.1, we will need to specify the partial derivatives up to the second order. To this end, we define an array $\left\{g_{n, k}(\theta)\right\}_{n \in \mathbb{N} ; k=1, \ldots, n}$ of random vectors in $\mathbb{R}^{4}$ by $g_{n, k}(\theta):=\nabla_{\theta}\left(l_{n, k}(\theta)\right)$, where

$$
g_{n, k}(\theta):=\left[\begin{array}{l}
g_{n, k}^{(1)} \\
g_{n, k}^{(2)} \\
g_{n, k}^{(3)} \\
g_{n, k}^{(4)}
\end{array}\right]:=\left[\begin{array}{l}
-\frac{1}{\alpha}-\frac{\Delta_{n} \beta \delta}{\alpha} \varepsilon_{n, k}+\frac{2 \Delta_{n} \delta}{\alpha} \varepsilon_{n, k} \operatorname{Im}\left(\frac{\Gamma^{\prime}\left(\Delta_{n} \delta\left(1+i \varepsilon_{n, k}\right)\right)}{\Gamma\left(\Delta_{n} \delta\left(1+i \varepsilon_{n, k}\right)\right)}\right) \\
\Delta_{n} \delta\left(\varepsilon_{n, k}-\tan (\beta / 2)\right) \\
2 \Delta_{n} \ln [2 \cos (\beta / 2)]-2 \Delta_{n} \frac{\Gamma^{\prime}\left(2 \Delta_{n} \delta\right)}{\Gamma\left(2 \Delta_{n} \delta\right)}+2 \Delta_{n} \operatorname{Re}\left(\frac{\Gamma^{\prime}\left(\Delta_{n} \delta\left(1+i \varepsilon_{n, k}\right)\right)}{\Gamma\left(\Delta_{n} \delta\left(1+i \varepsilon_{n, k}\right)\right)}\right) \\
-\Delta_{n} \frac{\beta}{\alpha}+\Delta_{n} \frac{2}{\alpha} \operatorname{Im}\left(\frac{\Gamma^{\prime}\left(\Delta_{n} \delta\left(1+i \varepsilon_{n, k}\right)\right)}{\Gamma\left(\Delta_{n} \delta\left(1+i \varepsilon_{n, k}\right)\right)}\right)
\end{array}\right] .
$$


(See Grigoletto and Provasi [3, Appendix A] for derivation of the gradient.) We also prepare the following asymptotics.

Lemma 3.2. It holds almost surely that

$$
\begin{aligned}
& \frac{\partial}{\partial \alpha} \operatorname{Im}\left(\frac{\Gamma^{\prime}\left(\Delta_{n} \delta\left(1+i \varepsilon_{n, 1}\right)\right)}{\Gamma\left(\Delta_{n} \delta\left(1+i \varepsilon_{n, 1}\right)\right)}\right)=-\frac{\Delta_{n} \delta \varepsilon_{n, 1}}{\alpha} \sum_{k=0}^{+\infty} \frac{\left(k+\Delta_{n} \delta\right)^{2}-\left(\Delta_{n} \delta \varepsilon_{n, 1}\right)^{2}}{\left(\left(k+\Delta_{n} \delta\right)^{2}+\left(\Delta_{n} \delta \varepsilon_{n, 1}\right)^{2}\right)^{2}} \\
& \sim-\frac{\Delta_{n} \delta \varepsilon_{n, 1}}{\alpha}\left(\frac{1}{\left(\Delta_{n} \delta\right)^{2}} \frac{1-\varepsilon_{n, 1}^{2}}{\left(1+\varepsilon_{n, 1}^{2}\right)^{2}}+\zeta(2)\right), \\
& \frac{\partial}{\partial \delta} \operatorname{Im}\left(\frac{\Gamma^{\prime}\left(\Delta_{n} \delta\left(1+i \varepsilon_{n, 1}\right)\right)}{\Gamma\left(\Delta_{n} \delta\left(1+i \varepsilon_{n, 1}\right)\right)}\right)=-2 \Delta_{n}^{2} \delta \varepsilon_{n, 1} \sum_{k=0}^{+\infty} \frac{k+\Delta_{n} \delta}{\left(\left(k+\Delta_{n} \delta\right)^{2}+\left(\Delta_{n} \delta \varepsilon_{n, 1}\right)^{2}\right)^{2}} \\
& \sim-2 \Delta_{n}^{2} \delta \varepsilon_{n, 1}\left(\frac{1}{\left(\Delta_{n} \delta\right)^{3}\left(1+\varepsilon_{n, 1}^{2}\right)^{2}}+\zeta(3)\right), \\
& \frac{\partial}{\partial \mu} \operatorname{Im}\left(\frac{\Gamma^{\prime}\left(\Delta_{n} \delta\left(1+i \varepsilon_{n, 1}\right)\right)}{\Gamma\left(\Delta_{n} \delta\left(1+i \varepsilon_{n, 1}\right)\right)}\right)=-\frac{\Delta_{n}}{\alpha} \sum_{k=0}^{+\infty} \frac{\left(k+\Delta_{n} \delta\right)^{2}-\left(\Delta_{n} \delta \varepsilon_{n, 1}\right)^{2}}{\left(\left(k+\Delta_{n} \delta\right)^{2}+\left(\Delta_{n} \delta \varepsilon_{n, 1}\right)^{2}\right)^{2}} \\
& \sim-\frac{\Delta_{n}}{\alpha}\left(\frac{1}{\left(\Delta_{n} \delta\right)^{2}} \frac{1-\varepsilon_{n, 1}^{2}}{\left(1+\varepsilon_{n, 1}^{2}\right)^{2}}+\zeta(2)\right), \\
& \frac{\partial}{\partial \alpha} \operatorname{Re}\left(\frac{\Gamma^{\prime}\left(\Delta_{n} \delta\left(1+i \varepsilon_{n, 1}\right)\right)}{\Gamma\left(\Delta_{n} \delta\left(1+i \varepsilon_{n, 1}\right)\right)}\right)=-\frac{2\left(\Delta_{n} \delta \varepsilon_{n, 1}\right)^{2}}{\alpha} \sum_{k=0}^{+\infty} \frac{k+\Delta_{n} \delta}{\left(\left(k+\Delta_{n} \delta\right)^{2}+\left(\Delta_{n} \delta \varepsilon_{n, 1}\right)^{2}\right)^{2}} \\
& \sim-\frac{2\left(\Delta_{n} \delta \varepsilon_{n, 1}\right)^{2}}{\alpha}\left(\frac{1}{\left(\Delta_{n} \delta\right)^{3}} \frac{1}{\left(1+\varepsilon_{n, 1}^{2}\right)^{2}}+\zeta(3)\right), \\
& \frac{\partial}{\partial \delta} \operatorname{Re}\left(\frac{\Gamma^{\prime}\left(\Delta_{n} \delta\left(1+i \varepsilon_{n, 1}\right)\right)}{\Gamma\left(\Delta_{n} \delta\left(1+i \varepsilon_{n, 1}\right)\right)}\right)=\Delta_{n} \sum_{k=0}^{+\infty} \frac{\left(k+\Delta_{n} \delta\right)^{2}-\left(\Delta_{n} \delta \varepsilon_{n, 1}\right)^{2}}{\left(\left(k+\Delta_{n} \delta\right)^{2}+\left(\Delta_{n} \delta \varepsilon_{n, 1}\right)^{2}\right)^{2}} \\
& \sim \Delta_{n}\left(\frac{1}{\left(\Delta_{n} \delta\right)^{2}} \frac{1-\varepsilon_{n, 1}^{2}}{\left(1+\varepsilon_{n, 1}^{2}\right)^{2}}+\zeta(2)\right), \\
& \frac{\partial}{\partial \mu} \operatorname{Re}\left(\frac{\Gamma^{\prime}\left(\Delta_{n} \delta\left(1+i \varepsilon_{n, 1}\right)\right)}{\Gamma\left(\Delta_{n} \delta\left(1+i \varepsilon_{n, 1}\right)\right)}\right)=-\frac{2 \Delta_{n}}{\alpha} \sum_{k=0}^{+\infty} \frac{k+\Delta_{n} \delta}{\left(\left(k+\Delta_{n} \delta\right)^{2}+\left(\Delta_{n} \delta \varepsilon_{n, 1}\right)^{2}\right)^{2}} \\
& \sim-\frac{2 \Delta_{n}}{\alpha}\left(\frac{1}{\left(\Delta_{n} \delta\right)^{3}} \frac{1}{\left(1+\varepsilon_{n, 1}^{2}\right)^{2}}+\zeta(3)\right),
\end{aligned}
$$

where all the asymptotics hold when $n \uparrow+\infty$.

Proof. The proof entails rather lengthy algebra of somewhat routine nature. To avoid overloading this proof, we only consider the first claim and omit all the rest. To this end, we have only to justify the interchange of the differentiation and the infinite sum as

$$
\frac{\partial}{\partial \alpha} \sum_{k=1}^{+\infty} \frac{1}{\left(k+\Delta_{n} \delta\right)^{2}+\left(\left(x_{n, 1}-\Delta_{n} \mu\right) / \alpha\right)^{2}}=\sum_{k=1}^{+\infty} \frac{\partial}{\partial \alpha} \frac{1}{\left(k+\Delta_{n} \delta\right)^{2}+\left(\left(x_{n, 1}-\Delta_{n} \mu\right) / \alpha\right)^{2}} .
$$

For convenience, we use the notation

$$
H(k ; \alpha):=\frac{1}{\left(k+\Delta_{n} \delta\right)^{2}+\left(\left(x-\Delta_{n} \mu\right) / \alpha\right)^{2}},
$$


with $x \in \mathbb{R}, \delta, \mu, \Delta_{n}$ fixed. It holds by the Taylor theorem that for $\lambda>0$ and for each $k \in \mathbb{N}$,

$$
\begin{aligned}
\left|\frac{H(k ; \alpha+\lambda)-H(k ; \alpha)}{\lambda}\right| & =\left|\int_{0}^{1} \frac{\partial}{\partial \alpha} H(k ; \alpha+\lambda s) d s\right| \\
& \leq \int_{0}^{1} \frac{2}{\alpha+\lambda s}\left(\frac{\left(x-\Delta_{n} \mu\right) /(\alpha+\lambda s)}{\left(k+\Delta_{n} \delta\right)^{2}+\left(\left(x-\Delta_{n} \mu\right) /(\alpha+\lambda s)\right)^{2}}\right)^{2} d s \\
& \leq \frac{2}{\alpha^{3}} \frac{\left(x-\Delta_{n} \mu\right)^{2}}{\left(k+\Delta_{n} \delta\right)^{4}} .
\end{aligned}
$$

Hence, we get

$$
\sum_{k=1}^{+\infty}\left|\frac{H(k ; \alpha+\lambda)-H(k ; \alpha)}{\lambda}\right| \leq \frac{2\left(x-\Delta_{n} \mu\right)^{2}}{\alpha^{3}} \sum_{k=1}^{+\infty} \frac{1}{\left(k+\Delta_{n} \delta\right)^{4}} \leq \frac{2\left(x-\Delta_{n} \mu\right)^{2}}{\alpha^{3}} \zeta(4),
$$

which justifies the interchange with the help of the dominated convergence theorem. The asymptotics are straightforward by splitting the sum into two parts $k=0$ and $k \geq 1$ with the help of the definition of the Riemann zeta function.

3) Note that using the expressions $\underline{3.5}$, we can show that

$$
\mathscr{I}(\boldsymbol{\theta})=\lim _{n \uparrow+\infty} \sum_{k=1}^{n} R_{n} \mathbb{E}_{\theta}\left[g_{n, k}(\theta) g_{n, k}(\theta)^{\top}\right] R_{n} .
$$

In order to complete the proof of Theorem 2.1, it suffices to prove the following two lemmas (see Section 4.1 in Kawai and Masuda [5] and the references therein for details).

Lemma 3.3. The symmetric matrix $\mathscr{I}(\theta)$ is well defined and is given by (2.2).

Lemma 3.4. (i) It holds that

$$
\lim _{n \uparrow+\infty} R_{n}\left(\sum_{k=1}^{n} \mathbb{E}_{\theta}\left[g_{n, k}(\theta)\right] \mathbb{E}_{\theta}\left[g_{n, k}(\theta)\right]^{\top}\right) R_{n}=0,
$$

where the right hand side indicates the zero matrix in $\mathbb{R}^{4 \times 4}$.

(ii) It holds that

$$
\lim _{n \uparrow+\infty} \sup _{\theta \in \Theta} \sum_{k=1}^{n}\left(\mathbb{E}_{\theta}\left[\left|R_{n} g_{n, k}(\theta)\right|^{4}\right]+\mathbb{E}_{\theta}\left[\left|R_{n} \operatorname{Hess}_{\theta}\left(l_{n, k}(\theta)\right) R_{n}\right|^{2}\right]\right)=0 .
$$

Note that (ii) in particular verifies the Lindeberg condition: for every $a>0$,

$$
\lim _{n \uparrow+\infty} \sum_{k=1}^{n} \mathbb{E}_{\theta}\left[\left|R_{n} g_{n, k}(\theta)\right|^{2} 1\left(\left|R_{n} g_{n, k}(\theta)\right| \geq a\right)\right]=0 .
$$

4) We here prove Lemma 3.3 Recall the definition of the digamma function

$$
\frac{\Gamma^{\prime}(z)}{\Gamma(z)}=-\frac{1}{z}-\gamma-\sum_{l=1}^{+\infty}\left(\frac{1}{l+z}-\frac{1}{l}\right), \quad z \in \mathbb{C}
$$

and also that for each $x>0$ and $y \in \mathbb{R}$,

$$
\begin{gathered}
\operatorname{Re}\left(\frac{\Gamma^{\prime}(x+i y)}{\Gamma(x+i y)}\right)=-\frac{x}{x^{2}+y^{2}}-\gamma-\sum_{l=1}^{+\infty}\left(\frac{l+x}{(l+x)^{2}+y^{2}}-\frac{1}{l}\right) \\
\operatorname{Im}\left(\frac{\Gamma^{\prime}(x+i y)}{\Gamma(x+i y)}\right)=\sum_{l=0}^{+\infty} \frac{y}{(l+x)^{2}+y^{2}}
\end{gathered}
$$


where the both infinite sums are well defined. Note that with respect to the variable $y$, the former is even, while the latter is odd. It is straightforward that as $n \uparrow+\infty$,

$$
-2 \Delta_{n} \frac{\Gamma^{\prime}\left(2 \Delta_{n} \delta\right)}{\Gamma\left(2 \Delta_{n} \delta\right)} \rightarrow \frac{1}{\delta},
$$

and that almost surely as $n \uparrow+\infty$,

$$
\begin{aligned}
& \operatorname{Re}\left(\frac{\Gamma^{\prime}\left(\Delta_{n} \delta\left(1+i \varepsilon_{n, 1}\right)\right)}{\Gamma\left(\Delta_{n} \delta\left(1+i \varepsilon_{n, 1}\right)\right)}\right)=-\frac{1}{\Delta_{n} \delta} \frac{1}{1+\varepsilon_{n, 1}^{2}}-\gamma-\sum_{l=1}^{+\infty}\left(\frac{l+\Delta_{n} \delta}{\left(l+\Delta_{n} \delta\right)^{2}+\left(\Delta_{n} \delta \varepsilon_{n, 1}\right)^{2}}-\frac{1}{l}\right) \sim-\frac{1}{\Delta_{n} \delta} \frac{1}{1+\varepsilon_{n, 1}^{2}}, \\
& \operatorname{Im}\left(\frac{\Gamma^{\prime}\left(\Delta_{n} \delta\left(1+i \varepsilon_{n, 1}\right)\right)}{\Gamma\left(\Delta_{n} \delta\left(1+i \varepsilon_{n, 1}\right)\right)}\right)=\frac{1}{\Delta_{n} \delta} \frac{\varepsilon_{n, 1}}{1+\varepsilon_{n, 1}^{2}}+\sum_{l=1}^{+\infty} \frac{\Delta_{n} \delta \varepsilon_{n, 1}}{\left(l+\Delta_{n} \delta\right)^{2}+\left(\Delta_{n} \delta \varepsilon_{n, 1}\right)^{2}} \sim \frac{1}{\Delta_{n} \delta} \frac{\varepsilon_{n, 1}}{1+\varepsilon_{n, 1}^{2}} .
\end{aligned}
$$

By using the above results, we get

$$
\begin{aligned}
& g_{n, 1}^{(1)}(\theta) \sim-\frac{1}{\alpha} \frac{1-\varepsilon_{n, 1}^{2}}{1+\varepsilon_{n, 1}^{2}}, \\
& g_{n, 1}^{(2)}(\theta)=\Delta_{n} \delta\left(\varepsilon_{n, 1}-\tan \frac{\beta}{2}\right), \\
& g_{n, 1}^{(3)}(\theta) \sim-\frac{1}{\delta} \frac{1-\varepsilon_{n, 1}^{2}}{1+\varepsilon_{n, 1}^{2}}, \\
& g_{n, 1}^{(4)}(\theta) \sim \frac{2}{\alpha \delta} \frac{\varepsilon_{n, 1}}{1+\varepsilon_{n, 1}^{2}},
\end{aligned}
$$

as $n \uparrow+\infty$. By denoting by $\mathscr{I}_{l_{1}, l_{2}}(\theta)$ the $\left(l_{1}, l_{2}\right)$-entry of $\mathscr{I}(\theta)$, we readily deduce by means of the LLN that

$$
\begin{aligned}
& \mathscr{I}_{1,1}(\theta)=\frac{1}{\alpha^{2}} \int_{\mathbb{R}}\left(\frac{1-x^{2}}{1+x^{2}}\right)^{2} \phi(x) d x=\frac{1}{2 \alpha^{2}}, \\
& \mathscr{I}_{2,2}(\theta)=\frac{\delta}{2(\cos (\beta / 2))^{2}}, \\
& \mathscr{I}_{3,3}(\theta)=\frac{1}{\delta^{2}} \int_{\mathbb{R}}\left(\frac{1-x^{2}}{1+x^{2}}\right)^{2} \phi(x) d x=\frac{1}{2 \delta^{2}}, \\
& \mathscr{I}_{4,4}(\theta)=\frac{4}{\alpha^{2} \delta^{2}} \int_{\mathbb{R}}\left(\frac{x}{1+x^{2}}\right)^{2} \phi(x) d x=\frac{1}{2 \alpha^{2} \delta^{2}}, \\
& \mathscr{I}_{1,2}(\theta)=\mathscr{I}_{2,1}(\theta)=0, \\
& \mathscr{I}_{2,3}(\theta)=\mathscr{I}_{3,2}(\theta)=0, \\
& \mathscr{I}_{3,4}(\theta)=\mathscr{I}_{4,3}(\theta)=-\frac{2}{\alpha \delta^{2}} \int_{\mathbb{R}} \frac{1-x^{2}}{1+x^{2}} \frac{x}{1+x^{2}} \phi(x) d x=0, \\
& \mathscr{I}_{1,3}(\theta)=\mathscr{I}_{3,1}(\theta)=\frac{1}{\alpha \delta} \int_{\mathbb{R}} \frac{1-x^{2}}{1+x^{2}} \frac{1-x^{2}}{1+x^{2}} \phi(x) d x=\frac{1}{2 \alpha \delta}, \\
& \mathscr{I}_{2,4}(\theta)=\mathscr{I}_{4,2}(\theta)=0, \\
& \mathscr{I}_{1,4}(\theta)=\mathscr{I}_{4,1}(\theta)=-\frac{2}{\alpha^{2} \delta} \int_{\mathbb{R}} \frac{1-x^{2}}{1+x^{2}} \frac{x}{1+x^{2}} \phi(x) d x=0 .
\end{aligned}
$$

This completes the proof of Lemma 3.3 .

5) It remains to prove Lemma 3.4 .

(i) With the help of Lemma 3.2 and the asymptotic behaviors of $g_{n, 1}(\theta)$ given in the proof of Lemma3.3 together with the bounded convergence theorem, it holds that as $n \uparrow+\infty$,

$$
\sqrt{n} R_{n} \mathbb{E}_{\theta}\left[g_{n, 1}(\theta)\right] \sim\left[\begin{array}{c}
-\frac{1}{\alpha} \int_{\mathbb{R}} \frac{1-x^{2}}{1+x^{2}} \phi(x) d x \\
0 \\
-\frac{1}{\delta} \int_{\mathbb{R}} \frac{1-x^{2}}{1+x^{2}} \phi(x) d x \\
\frac{2}{\alpha \delta} \int_{\mathbb{R}} \frac{x}{1+x^{2}} \phi(x) d x
\end{array}\right]=0,
$$


which is enough to prove the claim.

(ii) With the help of the asymptotic behaviors of $g_{n, 1}(\theta)$ given in the proof of Lemma 3.3 and notation $r_{n}^{(2)}:=1 / \sqrt{n \Delta_{n}}$ and $r_{n}^{(k)}:=1 / \sqrt{n}$ for $k=1,3,4$, it suffices to check that

$$
\begin{aligned}
\sum_{k=1}^{n} \mathbb{E}_{\theta}\left[\left|r_{n}^{(1)} g_{n, k}^{(1)}(\theta)\right|^{4}\right] & \sim \frac{1}{n \alpha^{4}} \int_{\mathbb{R}}\left(\frac{1-x^{2}}{1+x^{2}}\right)^{4} \phi(x) d x=\frac{3}{8 n \alpha^{4}}, \\
\sum_{k=1}^{n} \mathbb{E}_{\theta}\left[\left|r_{n}^{(2)} g_{n, 1}^{(2)}(\theta)\right|^{4}\right] & =\frac{1}{n \Delta_{n}} \frac{3 \delta \Delta_{n}+2-\cos \beta}{4 \delta^{3}(\cos (\beta / 2))^{4}}, \\
\sum_{k=1}^{n} \mathbb{E}_{\theta}\left[\left|r_{n}^{(3)} g_{n, 1}^{(3)}(\theta)\right|^{4}\right] & \sim \frac{1}{n^{2} \delta^{4}} \int_{\mathbb{R}}\left(\frac{1-x^{2}}{1+x^{2}}\right)^{4} \phi(x) d x=\frac{3}{8 n \delta^{4}}, \\
\sum_{k=1}^{n} \mathbb{E}_{\theta}\left[\left|r_{n}^{(4)} g_{n, 1}^{(4)}(\theta)\right|^{4}\right] & \sim \frac{1}{n^{2}}\left(\frac{2}{\alpha \delta}\right)^{4} \int_{\mathbb{R}}\left(\frac{x}{1+x^{2}}\right)^{4} \phi(x) d x=\frac{3}{8 n \alpha^{4} \delta^{4}},
\end{aligned}
$$

each of which tends to zero as $n \uparrow+\infty$. Thanks to the compactness of the set $\Theta$, it follows that

$$
\lim _{n \uparrow+\infty} \sup _{\theta \in \Theta} \sum_{k=1}^{n} \mathbb{E}_{\theta}\left[\left|R_{n} g_{n, k}(\theta)\right|^{4}\right]=0 .
$$

We can derive each entry of the Hessian matrix $\operatorname{Hess}_{\theta}\left(l_{n, k}(\theta)\right)$ of the likelihood as

$$
\begin{aligned}
\partial_{\alpha}^{2} l_{n, k}(\theta) & =\frac{1}{\alpha^{2}}+\frac{2 \beta \Delta_{n} \delta \varepsilon_{n, k}}{\alpha}-\frac{2\left(\Delta_{n} \delta \varepsilon_{n, k}\right)^{2}}{\alpha^{2}} \sum_{l=0}^{+\infty} \frac{3\left(l+\Delta_{n} \delta\right)^{2}+\left(\Delta_{n} \delta \varepsilon_{n, k}\right)^{2}}{\left(\left(l+\Delta_{n} \delta\right)^{2}+\left(\Delta_{n} \delta \varepsilon_{n, k}\right)^{2}\right)^{2}} \\
& \sim \frac{1}{\alpha^{2}} \frac{1-4 \varepsilon_{n, k}^{2}-\varepsilon_{n, k}^{4}}{\left(1+\varepsilon_{n, k}^{2}\right)^{2}}, \\
\partial_{\beta}^{2} l_{n, k}(\theta) & =-\Delta_{n} \frac{\delta}{2(\cos (\beta / 2))^{2}}, \\
\partial_{\delta}^{2} l_{n, k}(\theta) & =-4 \Delta_{n}^{2} \sum_{l=0}^{+\infty} \frac{1}{\left(l+2 \Delta_{n} \delta\right)^{2}}+2 \Delta_{n}^{2} \sum_{l=0}^{+\infty} \frac{\left(l+\Delta_{n} \delta\right)^{2}-\left(\Delta_{n} \delta \varepsilon_{n, k}\right)^{2}}{\left(\left(l+\Delta_{n} \delta\right)^{2}+\left(\Delta_{n} \delta \varepsilon_{n, k}\right)^{2}\right)^{2}} \\
& \sim-\frac{1}{\delta^{2}} \frac{1-4 \varepsilon_{n, k}^{2}-\varepsilon_{n, k}^{4}}{\left(1+\varepsilon_{n, k}^{2}\right)^{2}}, \\
\partial_{\mu}^{2} l_{n, k}(\theta) & =\frac{2 \Delta_{n}^{2}}{\alpha^{2}} \sum_{l=0}^{+\infty} \frac{-\left(l+\Delta_{n} \delta\right)^{2}+\left(\Delta_{n} \delta \varepsilon_{n, k}\right)^{2}}{\left(\left(l+\Delta_{n} \delta\right)^{2}+\left(\Delta_{n} \delta \varepsilon_{n, k}\right)^{2}\right)^{2}} \sim \frac{2}{\alpha^{2} \delta^{2}} \frac{-1+\varepsilon_{n, k}^{2}}{\left(1+\varepsilon_{n, k}^{2}\right)^{2}},
\end{aligned}
$$

and

$$
\begin{aligned}
& \partial_{\alpha} \partial_{\beta} l_{n, k}(\theta)=-\frac{\Delta_{n} \delta}{\alpha} \varepsilon_{n, k}, \\
& \partial_{\alpha} \partial_{\delta} l_{n, k}(\theta)=-\frac{4 \Delta_{n}}{\alpha} \sum_{l=0}^{+\infty} \frac{(l+\Delta \delta)\left(\Delta_{n} \delta \varepsilon_{n, k}\right)^{2}}{\left((l+\Delta \delta)^{2}+\left(\Delta \delta \varepsilon_{n, k}\right)^{2}\right)^{2}} \sim-\frac{4}{\alpha \delta}\left(\frac{\varepsilon_{n, k}}{1+\varepsilon_{n, k}^{2}}\right)^{2}, \\
& \partial_{\alpha} \partial_{\mu} l_{n, k}(\theta)=\frac{\beta \Delta_{n}}{\alpha^{2}}-\frac{4 \Delta_{n}}{\alpha^{2}} \sum_{l=0}^{+\infty} \frac{\Delta_{n} \delta \varepsilon_{n, k}\left(l+\Delta_{n} \delta\right)^{2}}{\left(\left(l+\Delta_{n} \delta\right)^{2}+\left(\Delta_{n} \delta \varepsilon_{n, k}\right)^{2}\right)^{2}} \sim-\frac{4}{\alpha^{2} \delta} \frac{\varepsilon_{n, k}}{\left(1+\varepsilon_{n, k}^{2}\right)^{2}}, \\
& \partial_{\beta} \partial_{\delta} l_{n, k}(\theta)=-\Delta_{n} \frac{\tan (\beta / 2)}{2} \\
& \partial_{\beta} \partial_{\mu} l_{n, k}(\theta)=-\Delta_{n} \frac{1}{\alpha}, \\
& \partial_{\delta} \partial_{\mu} l_{n, k}(\theta)=-\frac{4 \Delta_{n}^{2}}{\alpha} \sum_{l=0}^{+\infty} \frac{\left(l+\Delta_{n} \delta\right) \Delta_{n} \delta \varepsilon_{n, k}}{\left(\left(l+\Delta_{n} \delta\right)^{2}+\left(\Delta_{n} \delta \varepsilon_{n, k}\right)^{2}\right)^{2}} \sim-\frac{4}{\alpha \delta^{2}} \frac{\varepsilon_{n, k}}{\left(1+\varepsilon_{n, k}^{2}\right)^{2}},
\end{aligned}
$$


where all the asymptotics hold almost surely as $n \uparrow+\infty$. It is straightforward to deduce that as $n \uparrow+\infty$,

$$
\sup _{\theta \in \Theta} \sum_{k=1}^{n} \mathbb{E}_{\theta}\left[\left|R_{n} \operatorname{Hess}_{\theta}\left(l_{n, k}(\theta)\right) R_{n}\right|^{2}\right]=\left[\begin{array}{cccc}
O(1 / n) & O\left(\Delta_{n} / n\right) & O(1 / n) & O(1 / n) \\
O\left(\Delta_{n} / n\right) & O(1 / n) & O\left(\Delta_{n} / n\right) & O\left(\Delta_{n} / n\right) \\
O(1 / n) & O\left(\Delta_{n} / n\right) & O(1 / n) & O(1 / n) \\
O(1 / n) & O\left(\Delta_{n} / n\right) & O(1 / n) & O(1 / n)
\end{array}\right],
$$

where the squared norm inside the expectation are understood to be componentwise. The proof of Lemma 3.4 is complete.

\subsection{Proof of Proposition 2.2}

The mean of $X_{1}$ is given by $\mu_{0}(\theta):=\mu+\alpha \delta \tan (\beta / 2)$, so that we may write

$$
\mathbb{E}_{\theta}\left[e^{i y X_{1}}\right]=\exp \left[i y \mu_{0}(\theta)+\int_{\mathbb{R}_{0}}\left(e^{i y z}-1-i y z\right) g(z ; \theta) d z\right], \quad y \in \mathbb{R} .
$$

According to Sato [12, Theorem 33.1], for each $T>0$, the measures $\left.\mathbb{P}_{\theta_{1}}\right|_{\mathscr{F} T}$ and $\left.\mathbb{P}_{\theta_{2}}\right|_{\mathscr{F} T}$ are equivalent iff the following conditions are fulfilled:

(a) $g\left(z ; \theta_{2}\right)=\gamma\left(z ; \theta_{1}, \theta_{2}\right) g\left(z ; \theta_{1}\right)$ for some Borel function $\gamma\left(\cdot ; \theta_{1}, \theta_{2}\right): \mathbb{R} \rightarrow(0, \infty)$;

(b) $\mu_{0}\left(\theta_{2}\right)=\mu_{0}\left(\theta_{1}\right)+\int_{\mathbb{R}} z\left(\gamma\left(z ; \theta_{1}, \theta_{2}\right)-1\right) g\left(z ; \theta_{1}\right) d z$

(c) $\int_{\mathbb{R}}\left(1-\sqrt{\gamma\left(z ; \theta_{1}, \theta_{2}\right)}\right)^{2} g\left(z ; \theta_{1}\right) d z<+\infty$.

Hence, it suffices to show that these three conditions hold true iff $\alpha_{1} \delta_{1}=\alpha_{2} \delta_{2}$ and $\mu_{1}=\mu_{2}$.

Concerning the behaviors of the Lévy density $g(z ; \theta)$ near the origin and at infinity, in view of the series expansion $z / \sinh (z)=1-z^{2} / 6+O\left(z^{4}\right)$ as $|z| \rightarrow 0$, it is easy to see that the Lévy density $g(z ; \theta)$ admits the following expansion

$$
g(z ; \theta)=\frac{\alpha \delta}{\pi z^{2}}\left(1+\frac{\beta}{\alpha} z+O\left(z^{2}\right)\right)
$$

as $|z| \rightarrow 0$. Note that $\sinh (x)$ behaves like $e^{x} / 2$ as $x \uparrow+\infty$, while it behaves like $-e^{-x} / 2$ as $x \downarrow-\infty$. We thus get

$$
g(z ; \theta) \sim \begin{cases}2 \delta z^{-1} \exp \{-(\pi-\beta) z / \alpha\}, & z \uparrow+\infty \\ 2 \delta|z|^{-1} \exp \{-(\pi+\beta)|z| / \alpha\}, & z \downarrow-\infty\end{cases}
$$

In particular, (3.6) as well as the fact that $g(z ; \theta)>0$ for every $z \neq 0$ ensures (a); more specifically, the singularities of $g\left(z ; \theta_{1}\right)$ and $g\left(z ; \theta_{2}\right)$ at the origin are canceled out since $g\left(z ; \theta_{2}\right) / g\left(z ; \theta_{1}\right) \sim \alpha_{2} \delta_{2} /\left(\alpha_{2} \delta_{2}\right)$ as $|z| \rightarrow 0$.

We turn to (c) with $\gamma\left(z ; \theta_{1}, \theta_{2}\right)=g\left(z ; \theta_{2}\right) / g\left(z ; \theta_{1}\right)$. Due to (3.6) and (3.7), it holds that

$$
\begin{aligned}
& \left(1-\sqrt{\gamma\left(z ; \theta_{1}, \theta_{2}\right)}\right)^{2} g\left(z ; \theta_{1}\right)=\left(\sqrt{g\left(z ; \theta_{1}\right)}-\sqrt{g\left(z ; \theta_{2}\right)}\right)^{2} \\
& \quad \sim \begin{cases}\frac{1}{\pi|z|^{2}}\left(\left(\sqrt{\alpha_{1} \delta_{1}}-\sqrt{\alpha_{2} \delta_{2}}\right)^{2}+\left(\sqrt{\alpha_{1} \delta_{1}}-\sqrt{\alpha_{2} \delta_{2}}\right)\left(\beta_{1} \sqrt{\frac{\delta_{1}}{\alpha_{1}}}-\beta_{2} \sqrt{\frac{\delta_{2}}{\alpha_{2}}}\right) z+O\left(z^{2}\right)\right), & |z| \rightarrow 0 \\
C_{+} z^{-1} \exp \left(-q_{+} z\right), & z \uparrow+\infty \\
C_{-}|z|^{-1} \exp \left(-q_{-}|z|\right), & z \downarrow-\infty\end{cases}
\end{aligned}
$$

for some positive constants $C_{ \pm}$and $q_{ \pm}$, depending on $\left(\theta_{1}, \theta_{2}\right)$. Hence, (c) holds true iff $\alpha_{1} \delta_{1}=\alpha_{2} \delta_{2}$, which we will impose in the rest of this proof.

The remaining (b) is equivalent to

$$
\mu_{1}+\alpha_{1} \delta_{1} \tan \frac{\beta_{1}}{2}-\mu_{2}-\alpha_{2} \delta_{2} \tan \frac{\beta_{2}}{2}=\int_{\mathbb{R}_{0}}\left(\delta_{1} \frac{\exp \left(\beta_{1} z / \alpha_{1}\right)}{\sinh \left(\pi z / \alpha_{1}\right)}-\delta_{2} \frac{\exp \left(\beta_{2} z / \alpha_{2}\right)}{\sinh \left(\pi z / \alpha_{2}\right)}\right) d z
$$

In the case $\alpha_{1} \delta_{1}=\alpha_{2} \delta_{2}=: C>0$, the last display can be rewritten as

$$
\mu_{1}-\mu_{2}+C\left(\tan \frac{\beta_{1}}{2}-\tan \frac{\beta_{2}}{2} \int_{\mathbb{R}_{0}}\left(\frac{\exp \left(\beta_{1} z / \alpha_{1}\right)}{\alpha_{1} \sinh \left(\pi z / \alpha_{1}\right)}-\frac{\exp \left(\beta_{2} z / \alpha_{2}\right)}{\alpha_{2} \sinh \left(\pi z / \alpha_{2}\right)}\right) d z\right)=0 .
$$


We now show that the function

$$
f\left(\alpha_{1}, \beta_{1} ; \alpha_{1}, \beta_{2}\right):=\tan \frac{\beta_{1}}{2}-\tan \frac{\beta_{2}}{2}-\int_{\mathbb{R}_{0}}\left(\frac{\exp \left(\beta_{1} z / \alpha_{1}\right)}{\alpha_{1} \sinh \left(\pi z / \alpha_{1}\right)}-\frac{\exp \left(\beta_{2} z / \alpha_{2}\right)}{\alpha_{2} \sinh \left(\pi z / \alpha_{2}\right)}\right) d z \equiv 0,
$$

rendering that (b) holds true iff $\mu_{1}=\mu_{2}$, which completes the proof of the proposition. First, we observe that

$$
f\left(\alpha_{1}, 0 ; \alpha_{2}, 0\right)=\int_{\mathbb{R}_{0}}\left(\frac{1}{\alpha_{2} \sinh \left(\pi z / \alpha_{2}\right)}-\frac{1}{\alpha_{1} \sinh \left(\pi z / \alpha_{1}\right)}\right) d z \equiv 0,
$$

since the integrand is odd, continuous in $\mathbb{R}$, and exponentially decreasing as $|z| \uparrow+\infty$. Next, using the fact that the variance of $\operatorname{Meixner}\left(\alpha_{k}, \beta_{k}, \delta_{k}, \mu_{k}\right)$ equals $\int_{\mathbb{R}} z^{2} g\left(z ; \theta_{k}\right) d z$, we derive

$$
\frac{1}{\alpha_{k}^{2}} \int_{\mathbb{R}_{0}} z \frac{\exp \left(\beta_{k} z / \alpha_{k}\right)}{\sinh \left(\pi z / \alpha_{k}\right)} d z=\frac{1}{2 \cos ^{2}\left(\beta_{k} / 2\right)} .
$$

Hence, we get

$$
\frac{\partial}{\partial \beta_{1}} f\left(\alpha_{1}, \beta_{1} ; \alpha_{1}, \beta_{2}\right)=\frac{1}{2\left(\cos \left(\beta_{1} / 2\right)\right)^{2}}-\frac{1}{\alpha_{1}^{2}} \int_{\mathbb{R}_{0}} z \frac{\exp \left(\beta_{1} z / \alpha_{1}\right)}{\sinh \left(\pi z / \alpha_{1}\right)} d z \equiv 0
$$

and $\left(\partial / \partial \beta_{2}\right) f\left(\alpha_{1}, \beta_{1} ; \alpha_{1}, \beta_{2}\right) \equiv 0$ in a similar manner. These imply that $f\left(\alpha_{1}, \beta_{1} ; \alpha_{1}, \beta_{2}\right) \equiv 0$. The proof is complete.

\section{Acknowledgements}

Research of HM is supported in part by Grant-in-Aid for Young Scientists (B) Japan.

\section{References}

[1] Aït-Sahalia, Y. and Jacod, J. (2008) Fisher's information for discretely sampled Lévy processes, Econometrica, 76 727-761.

[2] Grigelionis, B. (1999) Processes of Meixner type, Lithuanian Mathematics Journal, 39(1) 33-41.

[3] Grigoletto, M., Provasi, C. (2009) Simulation and estimation of the Meixner distribution, Communications in Statistics - Simulation and Computation, 38(1) 58-77.

[4] Houdré, C., Kawai, R. (2007) On layered stable processes, Bernoulli, 13(1) 252-278.

[5] Kawai, R., Masuda, H., Local asymptotic normality for normal inverse Gaussian Lévy processes with high-frequency sampling, available at http://hdl.handle.net/2324/17018.

[6] Kawai, R., Parameter sensitivity estimation for Meixner distribution and Lévy processes, available at http://sites.google.com/site/reiichirokawai/

[7] Le Cam, L. (1960) Locally asymptotically normal families of distributions. Certain approximations to families of distributions and their use in the theory of estimation and testing hypotheses. Univ. California Publ. Statist. 3, 37-98.

[8] Le Cam, L., Yang, G. L. (1990) Asymptotics in Statistics. Some Basic Concepts. Second Edition, Springer Series in Statistics. SpringerVerlag, New York.

[9] Masuda, H. (2009) Notes on estimating inverse-Gaussian and gamma subordinators under high-frequency sampling, Annals of the Institute of Statistical Mathematics 61, 181-195.

[10] Masuda, H. (2009) Joint estimation of discretely observed stable Lévy processes with symmetric Lévy density, J. Japan Statist. Soc. 39, 49-75.

[11] Rosiński, J. (2007) Tempering stable processes, Stochastic Processes and their Applications, 117(6) 677-707.

[12] Sato, K. (1999) Lévy Processes and Infinitely Divisible Distributions, Cambridge University Press, Cambridge.

[13] Schoutens, W., Teugels, J. L. (1998) Lévy processes, polynomials and martingales, Communications in Statistics: Stochastic Models, 14, $335-349$.

[14] van der Vaart, A. W. (1998) Asymptotic Statistics, Cambridge University Press, Cambridge. 


\section{List of MI Preprint Series, Kyushu University}

The Global COE Program

Math-for-Industry Education \& Research Hub

MI

MI2008-1 Takahiro ITO, Shuichi INOKUCHI \& Yoshihiro MIZOGUCHI

Abstract collision systems simulated by cellular automata

MI2008-2 Eiji ONODERA

The intial value problem for a third-order dispersive flow into compact almost

Hermitian manifolds

MI2008-3 Hiroaki KIDO

On isosceles sets in the 4-dimensional Euclidean space

MI2008-4 Hirofumi NOTSU

Numerical computations of cavity flow problems by a pressure stabilized characteristiccurve finite element scheme

MI2008-5 Yoshiyasu OZEKI

Torsion points of abelian varieties with values in nfinite extensions over a padic field

MI2008-6 Yoshiyuki TOMIYAMA

Lifting Galois representations over arbitrary number fields

MI2008-7 Takehiro HIROTSU \& Setsuo TANIGUCHI

The random walk model revisited

MI2008-8 Silvia GANDY, Masaaki KANNO, Hirokazu ANAI \& Kazuhiro YOKOYAMA Optimizing a particular real root of a polynomial by a special cylindrical algebraic decomposition

MI2008-9 Kazufumi KIMOTO, Sho MATSUMOTO \& Masato WAKAYAMA

Alpha-determinant cyclic modules and Jacobi polynomials 
MI2008-10 Sangyeol LEE \& Hiroki MASUDA

Jarque-Bera Normality Test for the Driving Lévy Process of a Discretely Observed Univariate SDE

MI2008-11 Hiroyuki CHIHARA \& Eiji ONODERA

A third order dispersive flow for closed curves into almost Hermitian manifolds

MI2008-12 Takehiko KINOSHITA, Kouji HASHIMOTO and Mitsuhiro T. NAKAO

On the $L^{2}$ a priori error estimates to the finite element solution of elliptic problems with singular adjoint operator

MI2008-13 Jacques FARAUT and Masato WAKAYAMA

Hermitian symmetric spaces of tube type and multivariate Meixner-Pollaczek polynomials

MI2008-14 Takashi NAKAMURA

Riemann zeta-values, Euler polynomials and the best constant of Sobolev inequality

MI2008-15 Takashi NAKAMURA

Some topics related to Hurwitz-Lerch zeta functions

MI2009-1 Yasuhide FUKUMOTO

Global time evolution of viscous vortex rings

MI2009-2 Hidetoshi MATSUI \& Sadanori KONISHI

Regularized functional regression modeling for functional response and predictors

MI2009-3 Hidetoshi MATSUI \& Sadanori KONISHI

Variable selection for functional regression model via the $L_{1}$ regularization

MI2009-4 Shuichi KAWANO \& Sadanori KONISHI

Nonlinear logistic discrimination via regularized Gaussian basis expansions

MI2009-5 Toshiro HIRANOUCHI \& Yuichiro TAGUCHII

Flat modules and Groebner bases over truncated discrete valuation rings 
MI2009-6 Kenji KAJIWARA \& Yasuhiro OHTA

Bilinearization and Casorati determinant solutions to non-autonomous $1+1$ dimensional discrete soliton equations

\section{MI2009-7 Yoshiyuki KAGEI}

Asymptotic behavior of solutions of the compressible Navier-Stokes equation around the plane Couette flow

MI2009-8 Shohei TATEISHI, Hidetoshi MATSUI \& Sadanori KONISHI

Nonlinear regression modeling via the lasso-type regularization

MI2009-9 Takeshi TAKAISHI \& Masato KIMURA

Phase field model for mode III crack growth in two dimensional elasticity

MI2009-10 Shingo SAITO

Generalisation of Mack's formula for claims reserving with arbitrary exponents for the variance assumption

MI2009-11 Kenji KAJIWARA, Masanobu KANEKO, Atsushi NOBE \& Teruhisa TSUDA Ultradiscretization of a solvable two-dimensional chaotic map associated with the Hesse cubic curve

\section{MI2009-12 Tetsu MASUDA}

Hypergeometric T -functions of the q-Painlevé system of type $E_{8}^{(1)}$

MI2009-13 Hidenao IWANE, Hitoshi YANAMI, Hirokazu ANAI \& Kazuhiro YOKOYAMA A Practical Implementation of a Symbolic-Numeric Cylindrical Algebraic Decomposition for Quantifier Elimination

MI2009-14 Yasunori MAEKAWA

On Gaussian decay estimates of solutions to some linear elliptic equations and its applications

MI2009-15 Yuya ISHIHARA \& Yoshiyuki KAGEI

Large time behavior of the semigroup on $L^{p}$ spaces associated with the linearized compressible Navier-Stokes equation in a cylindrical domain 
MI2009-16 Chikashi ARITA, Atsuo KUNIBA, Kazumitsu SAKAI \& Tsuyoshi SAWABE Spectrum in multi-species asymmetric simple exclusion process on a ring

MI2009-17 Masato WAKAYAMA \& Keitaro YAMAMOTO

Non-linear algebraic differential equations satisfied by certain family of elliptic functions

MI2009-18 Me Me NAING \& Yasuhide FUKUMOTO

Local Instability of an Elliptical Flow Subjected to a Coriolis Force

MI2009-19 Mitsunori KAYANO \& Sadanori KONISHI

Sparse functional principal component analysis via regularized basis expansions and its application

MI2009-20 Shuichi KAWANO \& Sadanori KONISHI

Semi-supervised logistic discrimination via regularized Gaussian basis expansions

MI2009-21 Hiroshi YOSHIDA, Yoshihiro MIWA \& Masanobu KANEKO

Elliptic curves and Fibonacci numbers arising from Lindenmayer system with symbolic computations

MI2009-22 Eiji ONODERA

A remark on the global existence of a third order dispersive flow into locally Hermitian symmetric spaces

MI2009-23 Stjepan LUGOMER \& Yasuhide FUKUMOTO

Generation of ribbons, helicoids and complex scherk surface in laser-matter Interactions

MI2009-24 Yu KAWAKAMI

Recent progress in value distribution of the hyperbolic Gauss map

MI2009-25 Takehiko KINOSHITA \& Mitsuhiro T. NAKAO

On very accurate enclosure of the optimal constant in the a priori error estimates for $H_{0}^{2}$-projection 
MI2009-26 Manabu YOSHIDA

Ramification of local fields and Fontaine's property (Pm)

MI2009-27 Yu KAWAKAMI

Value distribution of the hyperbolic Gauss maps for flat fronts in hyperbolic three-space

MI2009-28 Masahisa TABATA

Numerical simulation of fluid movement in an hourglass by an energy-stable finite element scheme

MI2009-29 Yoshiyuki KAGEI \& Yasunori MAEKAWA Asymptotic behaviors of solutions to evolution equations in the presence of translation and scaling invariance

MI2009-30 Yoshiyuki KAGEI \& Yasunori MAEKAWA

On asymptotic behaviors of solutions to parabolic systems modelling chemotaxis

MI2009-31 Masato WAKAYAMA \& Yoshinori YAMASAKI

Hecke's zeros and higher depth determinants

MI2009-32 Olivier PIRONNEAU \& Masahisa TABATA

Stability and convergence of a Galerkin-characteristics finite element scheme of lumped mass type

MI2009-33 Chikashi ARITA

Queueing process with excluded-volume effect

MI2009-34 Kenji KAJIWARA, Nobutaka NAKAZONO \& Teruhisa TSUDA

Projective reduction of the discrete Painlevé system of type $\left(A_{2}+A_{1}\right)^{(1)}$

MI2009-35 Yosuke MIZUYAMA, Takamasa SHINDE, Masahisa TABATA \& Daisuke TAGAMI Finite element computation for scattering problems of micro-hologram using DtN map 
MI2009-36 Reiichiro KAWAI \& Hiroki MASUDA

Exact simulation of finite variation tempered stable Ornstein-Uhlenbeck processes

MI2009-37 Hiroki MASUDA

On statistical aspects in calibrating a geometric skewed stable asset price model

MI2010-1 Hiroki MASUDA

Approximate self-weighted LAD estimation of discretely observed ergodic OrnsteinUhlenbeck processes

MI2010-2 Reiichiro KAWAI \& Hiroki MASUDA

Infinite variation tempered stable Ornstein-Uhlenbeck processes with discrete observations

MI2010-3 Kei HIROSE, Shuichi KAWANO, Daisuke MIIKE \& Sadanori KONISHI Hyper-parameter selection in Bayesian structural equation models

MI2010-4 Nobuyuki IKEDA \& Setsuo TANIGUCHI

The Itô-Nisio theorem, quadratic Wiener functionals, and 1-solitons

MI2010-5 Shohei TATEISHI \& Sadanori KONISHI

Nonlinear regression modeling and detecting change point via the relevance vector machine

MI2010-6 Shuichi KAWANO, Toshihiro MISUMI \& Sadanori KONISHI

Semi-supervised logistic discrimination via graph-based regularization

MI2010-7 Teruhisa TSUDA

UC hierarchy and monodromy preserving deformation

MI2010-8 Takahiro ITO

Abstract collision systems on groups

MI2010-9 Hiroshi YOSHIDA, Kinji KIMURA, Naoki YOSHIDA, Junko TANAKA \& Yoshihiro MIWA

An algebraic approach to underdetermined experiments 
MI2010-10 Kei HIROSE \& Sadanori KONISHI

Variable selection via the grouped weighted lasso for factor analysis models

MI2010-11 Katsusuke NABESHIMA \& Hiroshi YOSHIDA

Derivation of specific conditions with Comprehensive Groebner Systems

MI2010-12 Yoshiyuki KAGEI, Yu NAGAFUCHI \& Takeshi SUDOU

Decay estimates on solutions of the linearized compressible Navier-Stokes equation around a Poiseuille type flow

MI2010-13 Reiichiro KAWAI \& Hiroki MASUDA

On simulation of tempered stable random variates

MI2010-14 Yoshiyasu OZEKI

Non-existence of certain Galois representations with a uniform tame inertia weight

MI2010-15 Me Me NAING \& Yasuhide FUKUMOTO

Local Instability of a Rotating Flow Driven by Precession of Arbitrary Frequency

MI2010-16 Yu KAWAKAMI \& Daisuke NAKAJO

The value distribution of the Gauss map of improper affine spheres

MI2010-17 Kazunori YASUTAKE

On the classification of rank 2 almost Fano bundles on projective space

MI2010-18 Toshimitsu TAKAESU

Scaling limits for the system of semi-relativistic particles coupled to a scalar bose field

MI2010-19 Reiichiro KAWAI \& Hiroki MASUDA

Local asymptotic normality for normal inverse Gaussian Lévy processes with high-frequency sampling

MI2010-20 Yasuhide FUKUMOTO, Makoto HIROTA \& Youichi MIE

Lagrangian approach to weakly nonlinear stability of an elliptical flow 
MI2010-21 Hiroki MASUDA

Approximate quadratic estimating function for discretely observed Lévy driven SDEs with application to a noise normality test

MI2010-22 Toshimitsu TAKAESU

A Generalized Scaling Limit and its Application to the Semi-Relativistic Particles System Coupled to a Bose Field with Removing Ultraviolet Cutoffs

MI2010-23 Takahiro ITO, Mitsuhiko FUJIO, Shuichi INOKUCHI \& Yoshihiro MIZOGUCHI Composition, union and division of cellular automata on groups

MI2010-24 Toshimitsu TAKAESU

A Hardy's Uncertainty Principle Lemma in Weak Commutation Relations of Heisenberg-Lie Algebra

MI2010-25 Toshimitsu TAKAESU

On the Essential Self-Adjointness of Anti-Commutative Operators

MI2010-26 Reiichiro KAWAI \& Hiroki MASUDA

On the local asymptotic behavior of the likelihood function for Meixner Lévy processes under high-frequency sampling 